\title{
Psychological Implications of Mandatory Testing for Severe Acute Respiratory Syndrome Coronavirus 2 During the Global COVID-19 Pandemic
}

\author{
Geoffrey W. Peitz ${ }^{\mathrm{a}, \mathrm{b}}$, Ali Seifia
}

During an admission for an elective neurosurgical procedure, a patient developed hypoxia, dry cough, high grade fever, and her chest computed tomography (CT) showed ground glass opacities. As coronavirus disease 2019 (COVID-19) was gaining prevalence in the community, her neurointensivist recommended testing for infection with severe acute respiratory syndrome coronavirus 2 (SARS-CoV-2). The possibility of becoming a patient under investigation and of having the disease caused the patient severe anxiety, and she refused the test. In an effort to better understand this sentiment and whether patients should have the option to refuse the test, the physician posted a description of the situation on a COVID-19 health care workers social media group, generating a lively discussion among a diverse group of people with various opinions all around the world. Posters supporting the right to refuse the test gave arguments rooted in the bioethical principles of autonomy and nonmaleficence. Meanwhile, posters opposing the right to refuse the test gave arguments rooted in societal beneficence and justice. This article aims to discuss the psychological and ethical impacts of forcing the patients to be tested for the COVID- 19.

The first documented case of COVID-19 in the USA was reported in a 35-year-old man from Snohomish County, Washington on January 20, 2020. Since then, more than 78 million Americans have been tested for COVID-19 with more than 6 million positive cases in the USA [1]. Widespread testing for the disease is considered essential for managing its spread, but there are ethical and psychosocial concerns for individuals being tested. Most patients feel that coerced COVID-19 testing violates patient autonomy. Autonomy is the value that grants patients the power to decide which diagnostic tests or treatments they receive. There is precedent for violating patient autonomy, especially in the decision to accept or refuse vaccinations when vaccination refusal poses a public health threat [2].

Manuscript submitted September 3, 2020, accepted September 9, 2020

Published online December 9, 2020

aDepartment of Neurosurgery, University of Texas Health Science Center at San Antonio, San Antonio, TX 78229, USA

${ }^{b}$ Corresponding Author: Geoffrey W. Peitz, Department of Neurosurgery, University of Texas Health Science Center at San Antonio, 7703 Floyd Curl Drive, San Antonio, TX 78229, USA. Email: peitz@uthscsa.edu

doi: https://doi.org/10.14740/jnr634
However, as Dr. Halpern emphasized, this is an era of growing regard for patient autonomy in which removing requirements for patient consent is not straightforward [3]. Furthermore, it is difficult to justify restricting a patient's right to refuse a treatment or test that has a significant risk of harm (i.e., when nonmaleficence is not upheld).

Besides the transient discomfort from swabbing, there is virtually no expected physical harm from the COVID-19 test. However, the test can have a negative impact financially. Even if there were no cost to the test itself, a positive test prevents a patient from working unless they have a job conducive to working from home. This results in loss of paid-time-off or loss of pay for people without paid-time-off. There may also be fear that a positive COVID-19 test increases a worker's chance of being laid off or furloughed. Besides the financial impact, a positive COVID-19 test, like human immunodeficiency virus (HIV), has a negative social stigma [4].Previous literature supports the idea that people diagnosed or even suspected of having HIV face discrimination and psychosocial issues [3, 5]. Although some may find it difficult to imagine COVID-19 carrying the same stigma, it is conceivable that people with COVID-19 would fear blame for not practicing rigorous hygiene or not adhering to social distancing. It is also possible that elderly or dependent patients would fear losing access to their caregivers or being displaced from home if they were to test positive for COVID-19. With all of these negative psychosocial factors compounding the physical risk of COVID-19, there is valid concern that the current pandemic will lead to increased psychopathology, especially post-traumatic stress disorder (PTSD), as seen in previous pandemics and disasters $[6,7]$. Coerced COVID-19 testing would limit patients' control over the situation, which could amplify the psychological impact.

Despite the potential negative psychosocial impact of COVID-19 testing, there are clear benefits to society that must be considered. Without a proven, targeted treatment for COVID-19, the individual benefit of being tested is small compared to the societal benefit. Except in the case of clinical trials, the treatments for COVID-19 are supportive and do not change with a positive test. However, a positive test does influence isolation and quarantine measures and encourages the use of personal protective equipment (PPE). By isolating a confirmed COVID-19 patient or patient under investigation, quarantining those who have already been in contact with the patient, and applying PPE to those who treat them, the rate of infection 
decreases. Thus, the whole community benefits. Whereas these measures can be applied when patients are suspected of having COVID-19 even without a confirmatory test, there are at least two reasons why testing is useful. There is concern that without a positive test, a patient with COVID-19 may not be obliged to self-isolate and would continue to spread the disease, similar to the example of "Typhoid Mary". As a healthy carrier of Salmonella typhi in 1906, her nickname of "Typhoid Mary" had become associated with the spread of disease, as many were infected due to her denial of being ill. Mary's case is a salient example of how the healthcare system may provoke negative social attitudes towards disease carriers [8]. However, if a trustworthy patient agrees to comply with self-isolation, there is not a strong ethical argument to force testing.

A second, more substantial reason why testing is beneficial to society ties in with the principle of justice and more specifically, fairness in distribution of limited resources. Exponential spread of COVID-19 has overwhelmed many healthcare systems and still poses this threat to many more. Lack of PPE is especially of concern because it affects healthcare workers who have not yet contracted the disease and still go home to their families. An individual seeking care for symptoms consistent with COVID-19 but refusing to be tested for the disease would require health care workers to use more PPE than would be necessary if the individual accepted testing and did not have COVID-19. Furthermore, a symptomatic patient refusing testing would be occupying a bed in a COVID-19 unit which could displace a patient actually having the disease to a non-COVID-19 unit, countering isolation efforts. Therefore, patients refusing COVID-19 testing may result in wasted PPE and COVID-19-unit capacity that otherwise could have been used to prevent further spread of the disease. As a testament to the level of concern about this issue, many social media posters suggested that complying with recommended COVID-19 testing be a requirement to receiving care in the hospital, but as Angotti et al have explained in the case of HIV, this would constitute coercion [5]. Therefore, the public health benefit of mandating COVID-19 testing for suspected patients seeking care must be balanced with the potential negative psychosocial impacts of testing.

In conclusion, the authors' opinion is that testing for SARS-CoV-2 should be mandatory during the pandemic to properly guide isolation and fairly distribute limited PPE and hospital space, thereby maximizing public health. However, autonomy and avoidance of negative psychosocial repercussions are valid reasons to allow patients to refuse testing, especially in communities where the health care system has not been overwhelmed or in situations where the patient is healthy enough to go home and reliable enough to self-isolate. In the scenario that prompted this discussion, the patient eventually agreed to be tested for COVID-19 after conversations with the medical team about the risks and benefits. In situations where the patient and medical team cannot reach an agreement, the authors suggest that the test only be forced if not testing the individual poses a substantial threat to public health and the hospital ethics committee is involved.

\section{Acknowledgments}

The authors would like to thank all of the social media users who replied to Dr. Seifi's post regarding patient refusal of COVID-19 testing and whose comments were the inspiration for this article.

\section{Financial Disclosure}

None to declare.

\section{Conflict of Interest}

None to declare.

\section{Author Contributions}

Both Geoffrey Peitz and Ali Seifi are involved in drafting and revising the manuscript.

\section{Data Availability}

The authors declare that data supporting the findings of this study are available within the article.

\section{References}

1. The COVID tracking project. https://covidtracking.com/ data. Accessed September 1, 2020.

2. Grzybowski A, Patryn RK, Sak J, Zagaja A. Vaccination refusal. Autonomy and permitted coercion. Pathog Glob Health. 2017;111(4):200-205.

3. Halpern SD. HIV testing without consent in critically ill patients. JAMA. 2005;294(6):734-737.

4. April MD. Rethinking HIV exceptionalism: the ethics of opt-out HIV testing in sub-Saharan Africa. Bull World Health Organ. 2010;88(9):703-708.

5. Angotti N, Dionne KY, Gaydosh L. An offer you can't refuse? Provider-initiated HIV testing in antenatal clinics in rural Malawi. Health Policy Plan. 2011;26(4):307-315.

6. Galea S, Merchant RM, Lurie N. The mental health consequences of COVID-19 and physical distancing: the need for prevention and early intervention. JAMA Intern Med. 2020;180(6):817-818.

7. Neria Y, Nandi A, Galea S. Post-traumatic stress disorder following disasters: a systematic review. Psychol Med. 2008;38(4):467-480.

8. Marineli F, Tsoucalas G, Karamanou M, Androutsos G. Mary Mallon (1869-1938) and the history of typhoid fever. Ann Gastroenterol. 2013;26(2):132-134. 